cularly clearly how the algorithm works: the mutual information can be large only if, first, the units convey information (that is, they behave nontrivially) and if, second, they respond similarly, so they share this information. The Hebb rule essentially imposes the first constraint alone. By adding the second constraint the new rule allows information to be thrown away when it is not shared by other patches.

Maximizing mutual information can also be interpreted as prediction, because each unit can be used to predict the behaviour of neighbouring patches. The notion of prediction is more general than that of stability: we can look for properties that predict future inputs, or predict one set of sensory data through another sensory modality. Prediction can help to complete or interpret missing data, and where prediction fails something interesting is likely to be happening. For example, places where disparity changes sharply will usually correspond to the edges of objects.

Neural networks are inspired by real neurons, but is there is any reverse flow of inspiration? Might a rule such as this operate in the brain? It seems unlikely that neurons compute something as mathematically complex as the ratio of variances, let alone the determinants which occur in the more general expression for more than two units. Furthermore, some of the difficulties of back propagation apply to the multilayer version of this algorithm, which must somehow feed back a complex error signal to earlier stages in the neural pathway. But it is important not to be too intimidated by the mathematical formulation. After all, principal component analysis, which in its standard form requires matrix inversion, might seem an unlikely operation for neurons to accomplish. Yet it can be carried out by suitably organized hebbian machinery. It seems likely, in fact, that there are natural ways for neurons to carry out Becker and Hinton's kind of analysis, or something very close to it, and this may provide another clue to help us explore synaptic learning rules in the brain.

Graeme Mitchison is in the Physiological Laboratory, University of Cambridge, Cambridge CB2 3EG, UK. Richard Durbin is in the MRC Laboratory of Molecular Biology. Hills Road, Cambridge CB2 2QH, UK.

1. Rumelhart, D. E., Hinton, G. E. \& Williams, R. J. Nature 323, 533-536 (1986).

2. Becker, S. \& Hinton, G. E. Nature 355, 161-163 (1992).

3. Hebb, D. O. The Organization of Behavior (Wiley, New York, 1949).

4. Oja, E. J. math. Biol. 15, 267-273 (1982)

5. Linsker, R. Computer 105-117 (March 1988)

6. Sanger, T. D. Neural Networks 2, 459-473 (1989).

6. Sanger, T. D. Neural Networks 2, 459-473 (1989).
7. Földiák, P. in Proc. Int. Joint. Conf. Neural Networks Vol. 1, 401-405 (IEEE, New York, 1989).

8. Hubel, D. H. \& Wiesel, T. N. J. Physiol. 160, 106-154 (1962).

\title{
Radio days of a remnant supernova
}

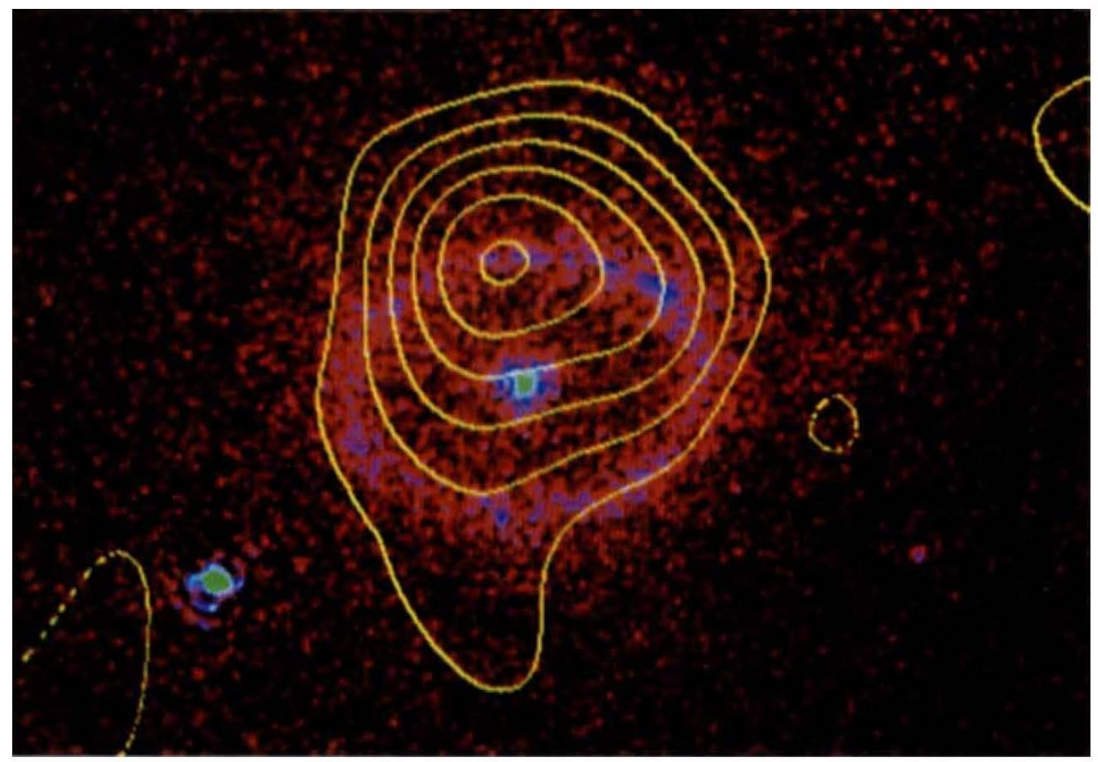

IN the history of supernova 1987A, now almost five years old, radioastronomers have so far had a negligible role. Apart from a brief initial outburst of radio emission, lasting no more than a few days, the expanding nebula set into motion by the explosion has been quite invisible at radio frequencies, and the steady thinning and cooling of the ejected material, and its interaction with the circumstellar material that surrounded the progenitor, has been followed largely through ultraviolet, optical and infrared observations. But elsewhere in this issue (Nature 355, 147149; 1992). L. Staveley-Smith et al. describe their detection of radio emission from the remnant, illustrated here overlayed on an optical picture from the Hubble Space Telescope. Evolution of the radio remnant over the coming years will provide a new tool to dissect the progress of the expanding remnant.

The key to understanding the remnant of SN1987A lies in the nature of its unusual progenitor star, which was first a red-giant, then a blue giant, before it exploded. In its red-giant phase, the star threw off a dense, slow-moving wind, which was succeeded by a more tenuous but faster wind from the blue-giant. The circumstellar material of the progenitor at the moment of explosion therefore consisted of a hot thin gas cocooned inside a cooler, thicker shell, with a shock wave created at the boundary as the blue-giant wind ran into the redgiant wind

The first brief flash of radio emission, reported by A. J. Turtle et al. (Nature 327, 38-40; 1987), was a very minor part of the initial supernova outburst, and was probably attributable to the propagation of the shock wave from the explosion through the thin material immediately surrounding what had been the progenitor star. According to R. A. Chevalier (Nature, in the press), the emission now detected by StaveleySmith and colleagues is due to the same expanding shock finally reaching the outer edges of the old blue-giant wind, just before it runs into the denser redgiant wind. Chevalier predicts that as the expanding ejecta passes through this boundary layer, the radio signal will rise and then diminish again, a signature which should be seen sometime during 1992.

After this transient appearance, SN1987A is unlikely to emerge as a fully formed radio supernova remnant for some time. The ages of radio remnants seen in other galaxies as well as our own are typically measured in hundreds of years at least, and there have been few opportunities for astronomers to observe a supernova at close enough hand to see the radio remnant arise from the expanding nebula. Before the advent of SN1987A, astronomers had to make do with studies of supernovae in other galaxies, and those that are detectable at radio frequencies have been either mature remnants or very new ones, which have faded within a few years.

Just before the radio recapture of SN1987A, however, J. Cowan, et al. (Astrophys. J. 379, L49-L51; 1991) spotted the reappearance of a 20-year old supernova, SN1970G in the galaxy M101, that had been radio-bright for about three years after outburst but which had then sunk below detectability. It is thought that the progenitor of SN1970G was, like that of SN1987A, a fairly massive star, and the explanation for the reappearance of radio emission from the former after 20 years and from the latter after five may be essentially the same.

David Lindley 